Historic, Archive Document

Do not assume content reflects current scientific knowledge, policies, or practices. 



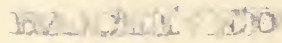 \\ SEP 81929 t

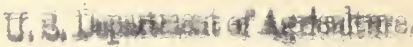

\section{WHOLESALE PRICE LIST OF PEONIES}

Fall of 1922 and Spring of 1923

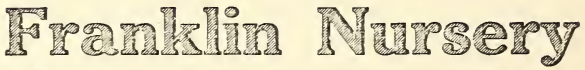

$1 /$ A. B. Franklin, Prop.

Minneapolis, Minn.

Prices quoted on peonies we offer are for strong divisions of from three to five eyes.

This list is for the Trade only and prices quoted are NET.

Six of one variety will be supplied at the dozen rate or twenty-five at the hundred rate.

If detailed description is desired, ask for our general catalogue which we will gladly mail upon request.

Unless credit already established or satisfactory bank references supplied, our terms are cash with order or before shipment is made.

No orders accepted for less than $\$ 5.00$.

We begin filling orders about September 1st, and continue shipments throughout the fall season.

All shipments made at purchaser's risk. We exercise great care in packing and with ordinary handling they should carry through to destination in fine condition.

We send out nothing but good, strong, healthy stock, true to name. 
Quality of stock taken into consideration, we believe our prices are as attractive as any offered.

Should you desire prices in quantity on varieties not listed herein, kindly write us and we will be pleased to quote on same if we have sufficient stock to warrant doing so. We devote several acres to the cultivation of the peony.

\section{Doz. 100}

1000

Achille (Calot) Delicate flesh....\$3.00 $\$ 20.00$ Alexander Dumas (Guerin)

Bright rose

$3.00 \quad 20.00$

Aurore (Dessert) White, $\$ 1$ ea...10.00

Bertha (Terry) Red...................... 4.50

Bernard De Pallisy (Crousse)

Blush pink

$3.00 \quad 22.50$

Bunch of Perfume (Kelway)

Salmon pink

$4.00 \quad 30.00$

Benjamin Franklin (Brand)

Bright crimson $\$ 1$ each

Chas. McKellip (Brand) Bright red, $\$ 2.50$ each.

Chestine Gowdy (Brand) Shell

pink with cream colored collar,

$\$ 2.50$ each

.25 .00

Duchess of Teck (Kelway)

Bright pink

Duchesse de Nemours (Calot)

White

25.00

Dr. H. Barnsby (Dessert) Deep

crimson, $\$ 4.00$ each.

$6.00 \quad 40.00$

$3.00 \quad 20.00$

Dorchester (Richardson) Hydrangea pink'

Edulis Superba (Lemon) Pink.... 2.50

Ella Adams. Light crimson...... 5.00

Ella Wheeler Wilcox (Brand)

Deep sea-shell pink $\$ 1$ each....10.00

Elwood Pleas (Pleas) Light rose shading to lilac, $\$ 5$ each. 50.00

Empress (Harrison) Soft pink.. $3.00 \quad 20.00$

Estafette (Dessert) Velvety pur-

ple with silvery border, $\$ 2.50$

each

25.00

Etta (Terry) Deep shell pink...... $5.00 \quad 35.00$ 
Eugene Bigot (Dessert) Brilliant

red, \$1 each.

10.00

Eugene Reignoux (Dessert) Semi

double. Brilliant carmine pink,

$\$ 1.50$ each

12.00

Eugenie Verdier (Calot) Pink... $6.00 \quad 45.00$

Festiva Maxima (Miellez) White $3.00 \quad 22.00$

Felix Crousse (Crousse) Brilliant red

Fragrans (Banks) Red................. 2.50

Francois Ortegat (Parmentier)

Red

$3.00 \quad 20.00$

Floral-Treasure (Rosenfield) Sal-

mon pink

$3.50 \quad 22.00$

Florence Nightingale (Brand)

White, each $\$ 1$..........................10.00

General Davoust (Crousse) Pink 2.50 20.00

Germaine Bigot (Dessert) Pale

lilac rose flecked crimson. $\$ 1$

each

10.00

Golden Wedding (Pleas) Yel-

lowish white, Center clear yellow

Golden Harvest (Rosenfield)......

Grandeur (Kelway) Light red....

Grandiffora Carnea Plena

(Lemon) Pink

$6.00 \quad 40.00$

$3.50 \quad 25.00$

$4.00 \quad 30.00$

Grandiflora Rosea (Guerin) Dark pink

$3.00 \quad 20.00 \$ 180.00$

$3.00 \quad 20.00$

Grover Cleveland (Terry) Bril-

liant crimson, $\$ 1.75$ each...........18.00 135.00

Humei (Anderson) Pink............. $2.00 \quad 15.00$

Fiarriet Farnsley (Brand) Soft

pink, $\$ 1$ each

.10 .00

Jenny Lind (Barr) Bright pink.. 3.5023 .00

L'Esperence (Calot) Pink.

$2.50 \quad 18.00$

La Tulipe (Calot) Blush white

with tulip like markings.

$\begin{array}{lll}3.00 & 20.00 \quad 180.00\end{array}$

Lady Lenora Bramwell (Kelway)

Syn. Dr. Bretonneau. Silvery

rose

$2.75 \quad 20.00$ 
Lady Alexandra Duff (Kelway)

Dox. 100

Charming pink, $\$ 5.50$ each......55.00

Limosel (Kelway) Bright lilac

rose

$3.50 \quad 25.00$

Lottie Collins (Kelway) Red...... $3.50 \quad 25.00$

Lora Dexheimer (Brand) Flaming crimson, $\$ 3.50$ each.

.35 .00

Lynda (Brand) Sulphur white.... 2.50

Marechal Vaillant (Calot) Red.. $3.00 \quad 20.00$

Marie Crousse (Crousse) Pale

lilac rose with salmon shades,

$\$ 1.75$ each

17.00

Marie Lemoine (Calot) White... 5.00

Mons. Paillet (Guerin) Light pink

Mme. Chaumy (Calot) Pink

$6.00 \quad 40.00$

$3.00 \quad 20.00$

Mme. Calot (Miellez) Flesh pink with shell pink shadings.

Mme. Courant (Courant) Flesh with yellow center

Mme. Crousse (Calot) Pure white

$3.00 \quad 20.00$

$2.75 \quad 20.00$

Mme. Breon (Guerin) Rosy flesh, center creamy white.

$4.00 \quad 30.00$

Mme. de Verneville (Crousse)

White

$3.50 \quad 25.00$

$3.00 \quad 20.00$

Nime. Ducel (Mechin) Silvery salmon

$4.50 \quad 32.00$

Mrs. Carew (Brand) Delicate silvery blush, $\$ 1.00$ each...........10.00 Octavie Demay (Calot) Pale pink

6.00

Princess Beatrice (Kelway) Pink guard petals center salmon and pink

$3.50 \quad 23.00$

Prince of Wales (Kelway) Shell pink, $\$ 1.25$ each. 12.00

Primavere (Lemoine) Nearest approach to yellow, $\$ 3.50$ ea...36.00 Princess Ellen (Terry) Light pink 



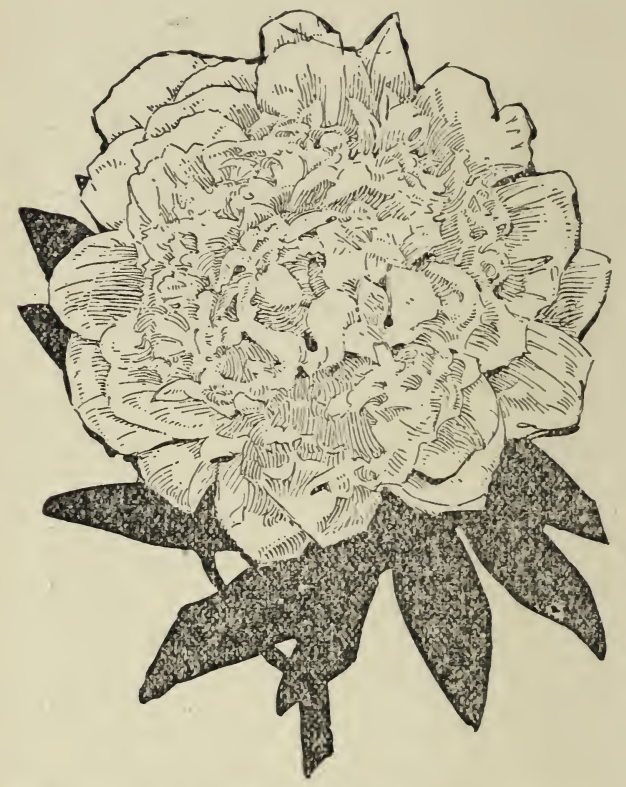

Fromklin Nureery 200 West Fifty-Eighth Street Minneapoli!s, Minm. 
Princess of Wales (Terry) Light pink

Doz. 100

Red Jacket (Harrison) red.

$3.50 \quad 25.00$

Reevesii. Dark purplish rose.... $\begin{array}{ll}6.00 & 40.00\end{array}$

Rosea Superba. Delicate rose $2.50 \quad 20.00$

Rubra Triumphans (Delache)

Crimson

$2.50 \quad 15.00$

Rubra Superba (Richardson)

Dark crimson

Stephanie (Terry) Pale rose,

nearly white, $\$ 2.00$ each..........20.00

Souvenir de l'Expcsition Uni-

verselle (Calot) Rosy pink..

$2.50 \quad 18.00$

Triomphe de l'Exrosition de

Lille (Calot) Pale hydrangea

pink

$3.00 \quad 20.00$

$4.50 \quad 35.00$

Tillie (Terry) Bright rose

$5.00 \quad 35.00$

$4.00 \quad 30.00$

The Bride (Terry) Lilac guards,

milk white center

$3.50 \quad 23.00$

Venus (Terry) Pale flesh.

$4.00 \quad 30.00$

Vicioire Modeste (Guerin) Deep

rose and chamois

$2.00 \quad 15.00$

Walter Morgan (Pleas) Pink

guards, yellow center.............25.00 175.00

Mixed. Our selection..................... $2.50 \quad 15.00 \quad 130.00$

In addition to growing a large acreage of peonies we grow large quantities of gladioli and will issue a list to the trade in early fall.

If smaller divisions are desired, kindly write us for prices.

Address all communications and send all remittances to the

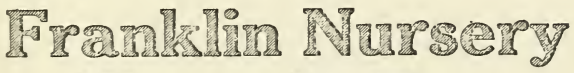

Minmeapolis, Minm。 
